\title{
Meta-analysis and systematic review of intravascular ultrasound versus angiography-guided drug eluting stent implantation in left main coronary disease in 4592 patients
}

\author{
Yue Wang ${ }^{1}$, Gary S. Mintz ${ }^{2}$, Zhichun Gu${ }^{3}$, Yue Qi ${ }^{4}$, Yue Wang ${ }^{1}$, Mengru Liu ${ }^{1}$ and Xiaofan Wu ${ }^{1 *}$ (D)
}

\begin{abstract}
Background: Although several meta-analyses have demonstrated the utility of intravascular ultrasound (IVUS) in guiding drug-eluting stent (DES) implantation compared to angiography-guidance, there has been a dearth of evidence in the left main coronary artery (LMCA) lesion subset.

Methods: We performed a meta-analysis to compare clinical outcomes of IVUS versus conventional angiography guidance during implantation of DES for patients with LMCA disease. Pubmed, Cochrane Library, Embase were searched.

Results: A total of 1002 publications were reviewed; and finally, seven clinical studies - one prospective randomized controlled trial and six observational studies with 4592 patients (1907 IVUS-guided and 2685 angiography-guided) were included in the meta-analysis. IVUS guidance was associated with a significant reduction in major adverse cardiac events (relative ratio [RR] 95\% Cl 0.61; 95\% confidence interval $[\mathrm{CI}] 0.53$ to $0.70 ; P<0.001$ ), all-cause death (RR 0.55; $95 \% \mathrm{Cl} 0.42$ to $0.71 ; P<0.001$ ), cardiac death (RR $0.45 ; 95 \% \mathrm{Cl} 0.32$ to $0.62 ; P<0.001$ ), myocardial infarction (RR 0.66; 95\% Cl 0.55 to 0.80; $P<0.001$ ), and stent thrombosis (RR $0.48 ; 95 \% \mathrm{Cl} 0.27$ to $0.84 ; P=0.01$ ) compared with angiographic guidance. However, there was no significant difference regarding target lesion revascularization (RR 0.60; $95 \% \mathrm{Cl} 0.31$ to $1.18 ; P=0.099$ ) and target vessel revascularization (RR $0.64 ; 95 \% \mathrm{Cl} 0.26$ to $1.56 ; P=0.322$ ).
\end{abstract}

Conclusions: Compared to angiographic guidance, IVUS-guided DES implantation was associated with better clinical outcomes for patients with LMCA lesions, especially hard endpoints of death, myocardial infarction, and stent thrombosis.

Keywords: Intravascular ultrasound, Angiography, Drug eluting stent, Left main disease, Meta-analysis

\section{Background}

Intravascular ultrasound (IVUS) has played a key role in contemporary stent-based percutaneous coronary interventions (PCI) by providing more detailed coronary anatomic information, assessing plaque burden accurately, selecting proper stent sizes, and optimizing stent expansion, apposition, and geographic miss [1-5]. One meta-analysis of seven randomized trials in the bare metal stent era [6]

\footnotetext{
* Correspondence: drwuxf@163.com

${ }^{1}$ Department of Cardiology, Beijing Anzhen Hospital, Capital Medical University, Beijing 100029, China

Full list of author information is available at the end of the article
}

and seven meta-analyses including both registries and randomized studies [7-13] in the drug-eluting stent (DES) era concluded that IVUS guidance improved patient outcomes compared to angiography guidance alone. However, and with one exception, these previously published meta-analyses did not address stent implantation to treat the subset of patients who present for PCI of left main coronary artery (LMCA) lesions; and there is increased interest in PCI intervention for LMCA disease since the results of two randomized DES versus bypass surgery studies - EXCEL and NOBLE - were recently presented $[14,15]$. Therefore, we performed the

(c) The Author(s). 2018 Open Access This article is distributed under the terms of the Creative Commons Attribution 4.0 International License (http://creativecommons.org/licenses/by/4.0/), which permits unrestricted use, distribution, and reproduction in any medium, provided you give appropriate credit to the original author(s) and the source, provide a link to the Creative Commons license, and indicate if changes were made. The Creative Commons Public Domain Dedication waiver (http://creativecommons.org/publicdomain/zero/1.0/) applies to the data made available in this article, unless otherwise stated. 
current meta-analysis of published studies comparing IVUS-guided versus angiography-guided DES implantation to treat LMCA lesions.

\section{Methods}

Data sources and search strategy

A computerized search was performed of Pubmed, Cochrane Library, Embase from January 1993 to October 2017. Mesh and combinations of the following terms were used in the search process: "ultrasonography, intravascular," "intravascular ultrasound," "intravascular ultrasoundguided," "IVUS," "IVUS-guided," "angiography," "angiography-guided," "left main coronary artery," "left main coronary stenosis," "left main coronary disease," "left main," "left main lesion," "LMCA," "drug-eluting stent," "sirolimus-eluting stent," "paclitaxel-eluting stent," "everolimus-eluting stent," "zotarolimus-eluting stent," "stent," and "DES." Two investigators (Yue Wang" and Yue Qi) independently screened the titles and abstracts and eventually examined the full texts of the original reports included in the study. Additional searches for potential studies were performed by reviewing the references of earlier meta-analyses concerning IVUS versus angiography-guided DES implantation. Complete data were retrieved from the studies for quantitative synthesis. In addition, studies with incomplete information - including abstracts of major meetings (Transcatheter Cardiovascular Therapeutics [TCT], Angioplasty Summit, American Heart Association [AHA], American College of Cardiology [ACC], EuroPCR, and World Congress of Cardiology [WCC]) and studies of IVUS versus angiography-guided DES implantation that included a subgroup of LMCA patients - were reviewed for other potentially relevant citations.

\section{Selection criteria}

Final inclusion of studies was based on the agreement of both reviewers. Randomized control trials (RCTs) and observational studies in English language were considered while studies in the non-English language literature were not included. Studies met the following pre-specified criteria: 1) clinical studies published in peer-reviewed journals with fully available data; 2) studies that included a comparison of IVUS-guided versus angiography-guided PCI with DES in LMCA lesions; and 3) follow-up time of at least 12 months. Reports of mixed treatment with bare-metal stent and DES implantation without separate clinical outcomes for the DES subgroup were excluded. Within this study, [16] patients $(n=1899)$ predominantly underwent DES implantation, with BMS implanted in only a small proportion of the study population (IVUS-guided arm: 1.3\%, angiography-guided arm: $3.0 \%)$. It was therefore deemed appropriate to include this study in the meta-analysis. In addition, studies with incomplete data were reviewed and later discussed, but not included in the formal meta-analysis.

\section{Endpoints and definition}

The primary endpoint in this meta-analysis was major adverse cardiac events (MACE), defined as the composite of death, MI (myocardial Infarction), and repeat revascularization. The secondary endpoint was all-cause death, cardiac death, MI, target lesion revascularization (TLR), target vessel revascularization (TVR) and stent thrombosis (ST; included definite, probable or possible $\mathrm{ST}$ ) according to the definition of the Academic Research Consortium [17].

\section{Data extractions}

We extracted DES data exclusively, thereby excluding bare metal stent data. The study's first author's name, publication date, study design, and follow-up duration; baseline clinical, angiographic, and procedural characteristics; and clinical outcomes were systematically reviewed and recorded by the same two reviewers (Yue Wang" and Yue Qi). Disagreements were resolved by discussion between them.

\section{Quality assessments}

The methodological quality of RCTs was assessed by the Cochrane Collaboration Risk of Bias tool [18]. The methodological quality of observational studies was assessed by the Newcastle-Ottawa scale (NOS) that consists of three factors: patient selection, comparability of the study groups, and assessment of outcomes [19]. A score of $0-9$ was allocated to each study except RCTs. Observational studies achieving six or more scores were considered to be of high quality [20].

\section{Statistical analyses}

Baseline characteristics between IVUS-guided versus angiography-guided groups were analyzed and compared, with mean $\pm S D$ for continuous variables using two-sample student's unpaired t-test and proportions for categorical variables using chi-square statistics. Acrossstudy summary relative ratios (RRs) with $95 \%$ confidence interval (CI) were produced to assess the efficacy of IVUS versus angiography guidance on adverse clinical events. The statistical heterogeneity between trials was assessed with chi-square tests and $\mathrm{I}^{2}$ statistics. When the $p$ value of chi-square test was $<0.10$ and/or the $\mathrm{I}^{2}$ was $\geq 50 \%$, significant heterogeneity was considered and a random-effects model would be selected. If not, the fixed-effects model was used instead. Egger's linear regression analysis was performed to quantitatively assess the underlying publication bias across the studies. In order to evaluate the stability and reliability of the primary endpoint result, we performed a sensitivity analysis 
of MACE by omitting each individual study in turn. All reported $p$-values were 2 -sided, and $P<0.05$ was considered to indicate statistical significance. All statistical analyses were performed using STATA 14.0 (Stata Corp, College Station, TX, USA).

\section{Results}

\section{Studies included}

A total of 1002 publications were reviewed, and 801 citations were screened by checking the title or abstract. Of these, 49 studies were reviewed in detail; and seven clinical studies (4592 patients) were included in the current formal meta-analysis, [16, 21-26] in which 1907 patients underwent IVUS-guided PCI and 2685 underwent angiography-guided PCI with DES implantation. (Fig. 1) One study was a RCT, [25] and the other six studies were observational registries [16, 21-24, 26]. Four studies performed propensity score matching $[16,22,23,26]$. As specified in the Methods, one meeting abstract published in 2016 (Effectiveness and safety of intravascular ultrasound guidance on clinical outcomes following drug-eluting stent implantation in unprotected left main coronary artery) and one study [27] that included LMCA subgroup analysis were reviewed in the Discussion, not included in our meta-analysis.

\section{Study characteristics}

Baseline characteristics of the included studies have been shown in Table 1 . The follow-up time ranged from 12 to 36 months. Of the seven studies, the follow-up time in four was 36 months. There were no statistically significant differences in the baseline characteristics between the IVUS-guided versus the angiography-guided groups, except for age, left ventricular ejection fraction and previous PCI. Table 2 demonstrating angiographic and procedural characteristics. Four studies mainly used first generation DES [21, 22, 24, 25], and both first and second-generation DES were implanted in two studies $[16,26]$. However, one study did not report the type of DES [23]. Most studies were frequent use of a two-stent technique except for one study by Kim [26].

\section{Assessment of quality}

Table 3 presented quality assessment results of included observational trials. Among the six observation studies, all had a scoring of $\geq 6$. The summary risk of bias of the RCT study was low. All studies were considered to be of high quality.

\section{Clinical outcomes}

Analyses for clinical outcomes have been presented in Fig. 2. The definition of MACE was slightly different across studies; two studies included cardiac death, $[21,25]$ and the other five included all-cause deaths [16, 21-24, 26]. Three studies reported TVR, [21, 22, 24] two reported TLR, [23, 25] and one study reported any revascularization not restricted to the target lesion [26] (Table 4).

MACE was reported in all seven studies (Table 5), the summary RR was $0.61(95 \%$ CI 0.53 to $0.70 ; P<0.001)$ in

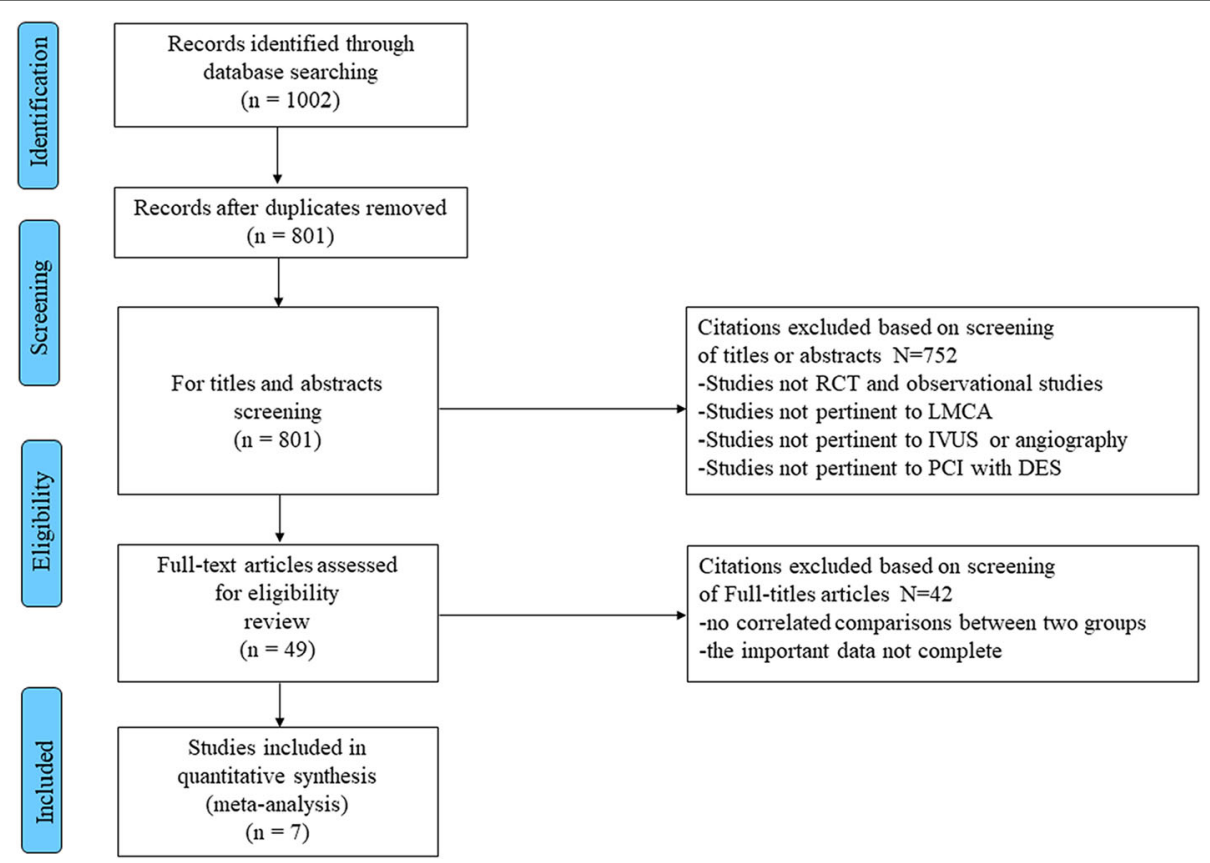

Fig. 1 Flow diagram of the process followed to identify the relevant studies that were included in the present meta-analysis 


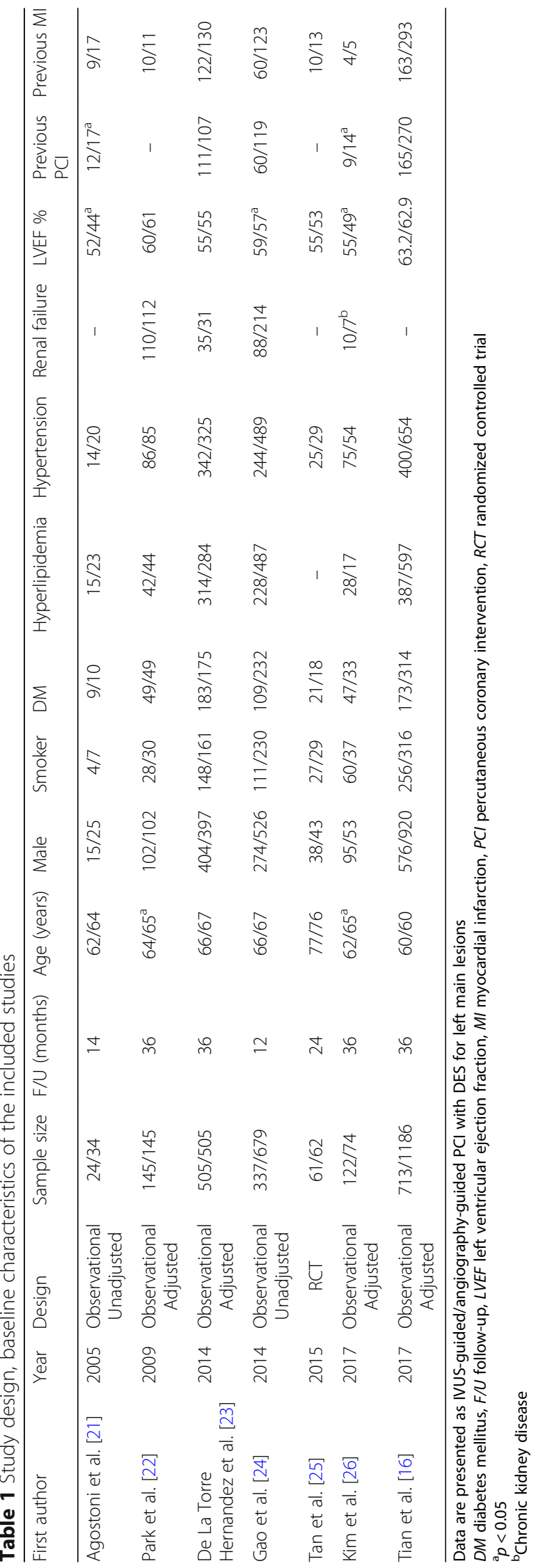




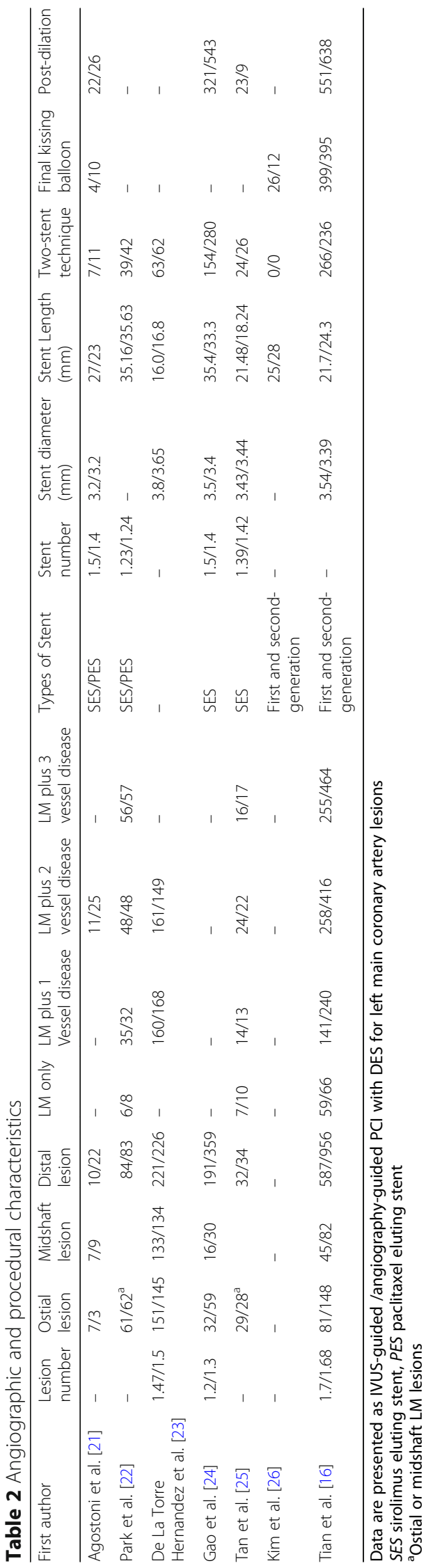




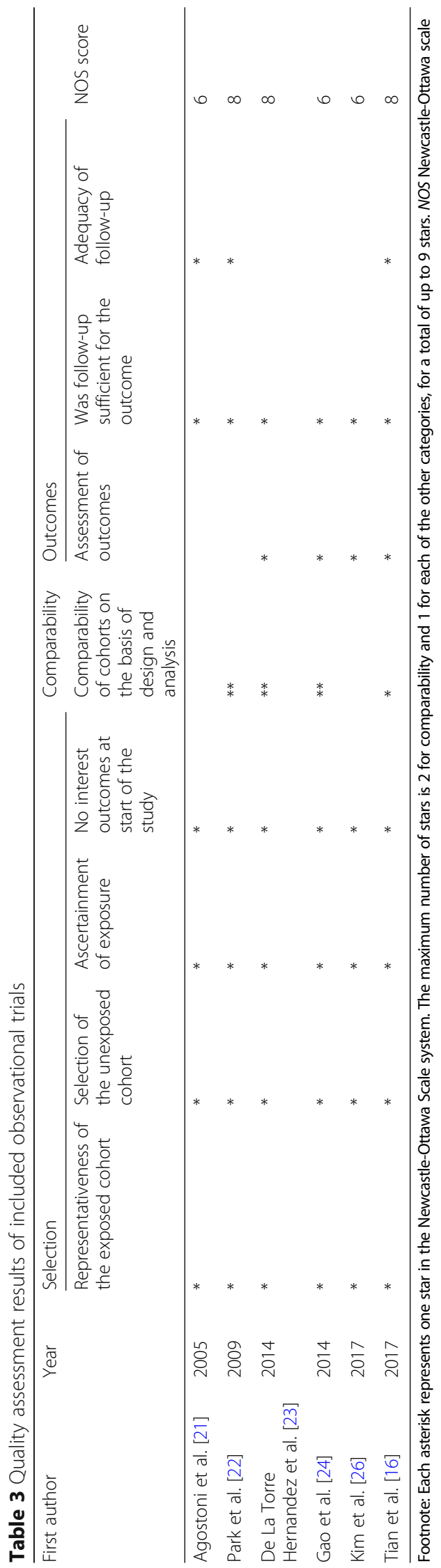




\section{a}

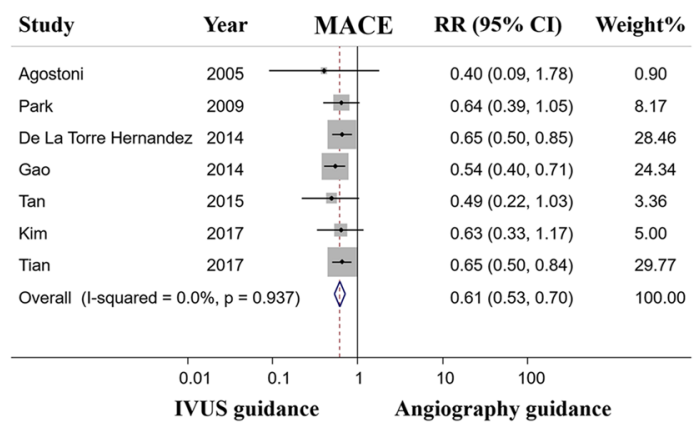

C

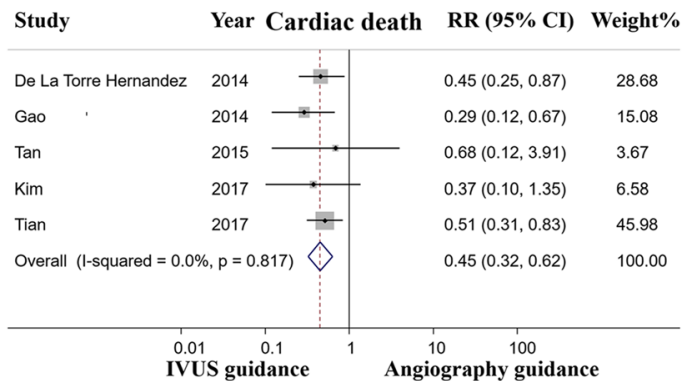

e

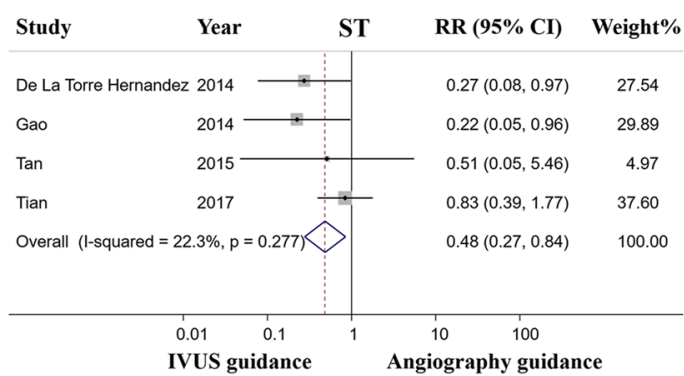

b

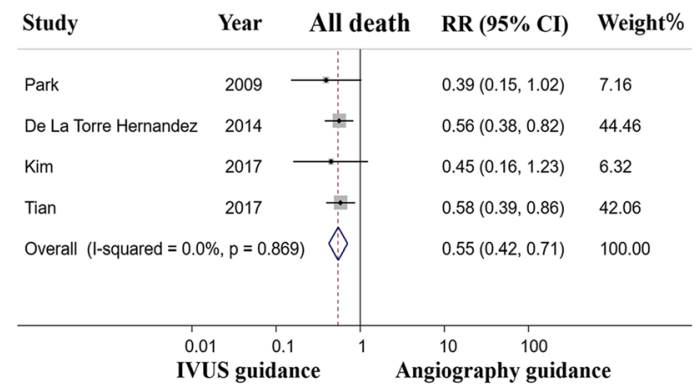

d

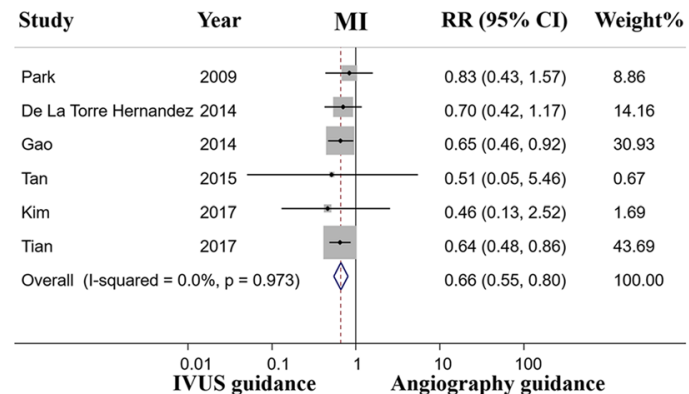

f

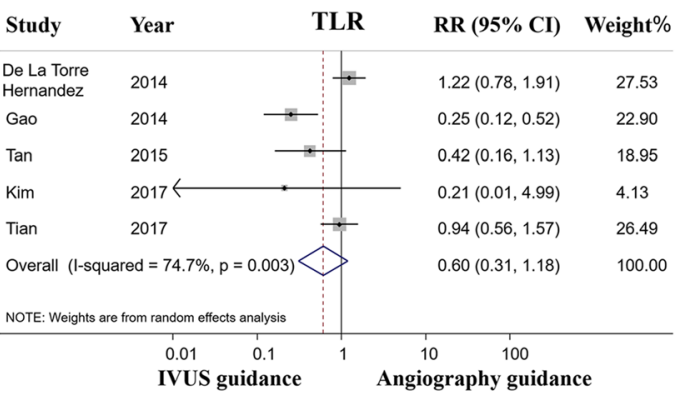

g

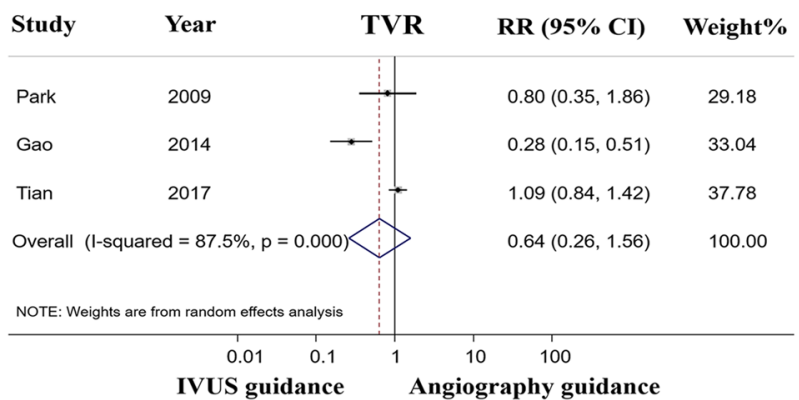

Fig. 2 Forest plot of RR for MACE (a), all cause death (b), cardiac death (c), MI (d), ST (e), TLR (f) and TVR (g) associated with IVUS guided vs angiography guided DES implantation to treat LMCA disease. Squares is the effect size of the individual studies; diamonds, the summarized effect size; horizontal lines, upper and lower border of 95\% confidence interval. DES = drug eluting stent; LMCA = left main coronary artery; IVUS = intravascular ultrasound; $\mathrm{MACE}=$ major adverse cardiac event; $\mathrm{Ml}=$ myocardial infarction; relative ratio $=\mathrm{RR} ; \mathrm{ST}=$ stent thrombosis; $\mathrm{TLR}=$ target lesion revascularization; TVR $=$ target vessel revascularization 
Table 4 The endpoint events and definitions

\begin{tabular}{llll}
\hline First author & MACE & Repeat revascularization & Stent thrombosis \\
\hline Agostoni et al. [21] & Cardiac death, MI, TVR & - & - \\
Park et al. 22] & All cause death, MI, TVR & TVR & - \\
De La Torre Hernandez et al. [23] & All cause death, Ml, TLR & TLR & Definite, probable \\
Gao et al. [24] & All cause death, MI, TVR & TLR, TVR & Definite, probable, possible \\
Tan et al. [25] & Cardiac death, MI, TLR & TLR & Definite, probable \\
Kim et al. [26] & All cause death, MI, TVR, TLR & TLR, TVR & - \\
Tian et al. [16] & All cause death, Ml & TLR, TVR & Definite, probable \\
\hline
\end{tabular}

MACE major adverse cardiovascular event, MI myocardial infarction, TLR target lesion revascularization, TVR target vessel revascularization

favor of IVUS-guided DES implantation. No evidence of statistical heterogeneity was noted among the included studies $\left(I^{2}=0 \% ; P=0.937\right)$ (Fig. 2a).

Four studies reported all-cause death and indicated that IVUS-guided DES implantation was associated with a significant reduction of all-cause mortality (RR $0.55 ; 95 \%$ CI 0.42 to $0.71 ; P<0.001$ ) with no statistical heterogeneity across the studies $\left(I^{2}=0 \% ; P=0.869\right)$ (Fig. 2b).

Of the seven studies, five were included in the analysis of cardiac death; the risk of cardiac death was significantly lower with IVUS guidance (RR $0.45 ; 95 \%$ CI 0.32 to $0.62 ; P<0.001)$ with no statistically significant heterogeneity $\left(I^{2}=0 \% ; P=0.817\right)$ (Fig. 2c).

Six studies were applied to the analysis of MI. The result was significantly in favor of IVUS-guided DES implantation (RR 0.66; 95\% CI 0.55 to $0.80 ; P<0.001$ ) with no heterogeneity $\left(I^{2}=0 \% ; P=0.973\right)$ (Fig. $\left.2 \mathrm{~d}\right)$.

Data on ST was reported in four studies. In PCI with DES implantation, IVUS-guidance markedly lowered the risk of definite/probable ST compared with the angiography guidance group (RR $0.48 ; 95 \%$ CI 0.27 to 0.84 ; $P=0.01)$, again with no statistical heterogeneity $\left(I^{2}=\right.$ 22.3\%; $P=0.277$ ) (Fig. 2e).

Six studies reported data regarding TLR and three reported TVR. Due to significant heterogeneities (TLR $I^{2}=74.7 \% ; P=0.003$, and TVR $\left.I^{2}=87.5 \% ; P<0.001\right)$, random effects models were used to estimate the summary effect of all studies. There were no significant statistical differences regarding TLR (RR $0.60 ; 95 \%$ CI 0.31 to $1.18 ; P=0.099$ ) and TVR (RR $0.64 ; 95 \%$ CI 0.26 to $1.56 ; P=0.322$ ) between the two groups (Fig. $2 \mathrm{f}$ and Fig. $2 \mathrm{~g}$ ).

\section{Sensitivity analysis}

Sensitivity analysis regarding the primary endpoint MACE has been presented in Fig. 3. After removing each study in sequence, the results were not statistically different from the summary RR across the seven studies, further indicating that IVUS-guided DES implantation was associated with a significant reduction in MACE.

\section{Publication bias}

Because only seven clinical studies were included the current meta-analysis, we assessed the asymmetry of publication using quantitative tools. No significant evidence of publication bias $(P=0.401$ for MACE, $P=0.058$ for all death, $P=0.709$ for cardiac death, $P=0.842$ for MI, $P=0.338$ for ST and $P=0.75$ for TLR) were observed on the basis of Egger's test (Fig. 4).

\section{Discussion}

This meta-analysis of seven studies consisting of 4592 patients demonstrated that IVUS-guided DES implantation when treating LMCA lesions was associated with a significantly reduced risk of MACE, all-cause death,

Table 5 Clinical outcomes for MACE in the IVUS-guided and angiography-guided groups

\begin{tabular}{|c|c|c|c|c|c|c|c|}
\hline First author & Year & Sample size & IVUS guidance ${ }^{a}$ & Angiography guidance $^{a}$ & RR & $95 \% \mathrm{Cl}$ & $P$-value \\
\hline Agostoni et al. [21] & 2005 & $24 / 34$ & $8 \%$ & $20 \%$ & 0.4 & $0.09-1.78$ & 0.18 \\
\hline Park et al. [22] & 2009 & $145 / 145$ & - & - & 0.64 & $0.39-1.05$ & 0.074 \\
\hline De La Torre Hernandez et al. [23] & 2014 & $505 / 505$ & $14.4 \%$ & $22.2 \%$ & 0.65 & $0.5-0.85$ & 0.006 \\
\hline Gao et al. [24] & 2014 & $337 / 679$ & $14.8 \%$ & $27.7 \%$ & 0.54 & $0.4-0.71$ & $<0.001$ \\
\hline Tan et al. [25] & 2015 & $61 / 62$ & $13.1 \%$ & $27.4 \%$ & 0.49 & $0.22-1.03$ & 0.031 \\
\hline Kim et al. [26] & 2017 & $122 / 74$ & $21 \%$ & $43 \%$ & 0.63 & $0.33-1.17$ & 0.149 \\
\hline Tian et al. [16] & 2017 & $713 / 1186$ & $5.3 \%$ & $8.1 \%$ & 0.65 & $0.5-0.84$ & 0.001 \\
\hline
\end{tabular}

$C l$ confidence interval, MACE major adverse cardiac events, $R R$ relative ratio

${ }^{a}$ Percentage of total population 


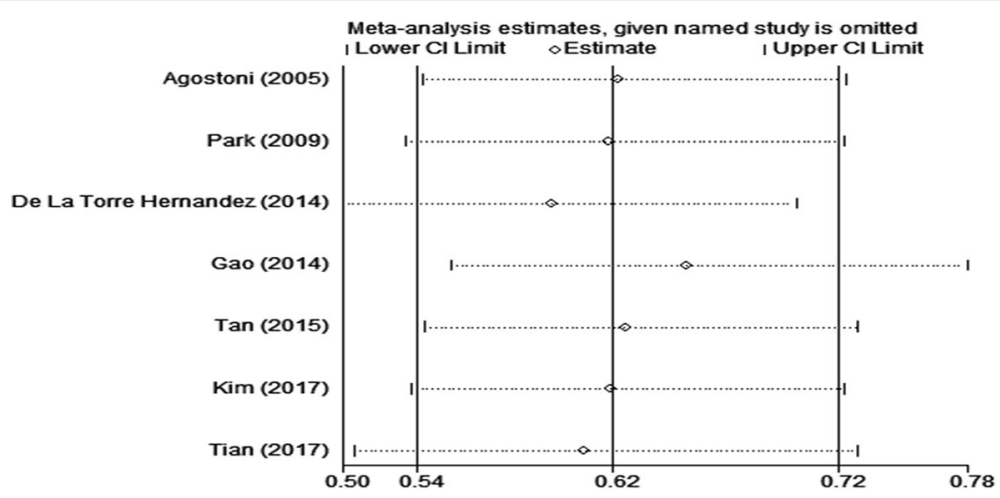

Fig. 3 Sensitivity analysis of MACE by omitting each individual study in turn. Cycle is relative ratio; horizontal lines, upper and lower border of $95 \%$ confidence interval. MACE $=$ major adverse cardiac event

cardiac death, MI, and ST, when compared with angiography-guidance.

Using coronary angiography, LMCA lesions may be obscured by overlapping vessels, streaming of injected contrast, and lack of a normal reference segment that limits the utility of angiography in determining lesion severity and selecting treatment strategy [28]. A study by Chakrabarti demonstrated that $11.2 \%$ (17 of 152) patients with LMCA disease by core laboratory assessment were deemed normal by clinical assessment in the National Cardiovascular Data Registry (NCDR), whereas 56.7\% (177 of 312) patients having significant LMCA disease by clinical assessment in the NCDR had no LMCA lesions by core laboratory analysis [29]. In addition, visual inspection of the angiogram may result in an incorrect diagnosis of LMCA disease severity. In one study including 213 patients with LMCA stenosis, $23 \%$ of patients had a diameter stenosis $<50 \%$ on the angiogram while the FFR was $<0.80$, who actually had hemodynamically significant stenosis and needed revascularization [30].

Conversely, IVUS can detect significant narrowing and assess angiographically ambiguous LMCA lesions; [31] and IVUS correlates with FFR [32]. IVUS has been used to guide decision-making with regards to PCI or bypass surgery in patients with LMCA disease [33]. IVUS also has the ability to assess LMCA plaque distribution and predict hemodynamically significant jailing of the left circumflex after single stent cross-over in a manner not possible using coronary angiography [34, 35]. More

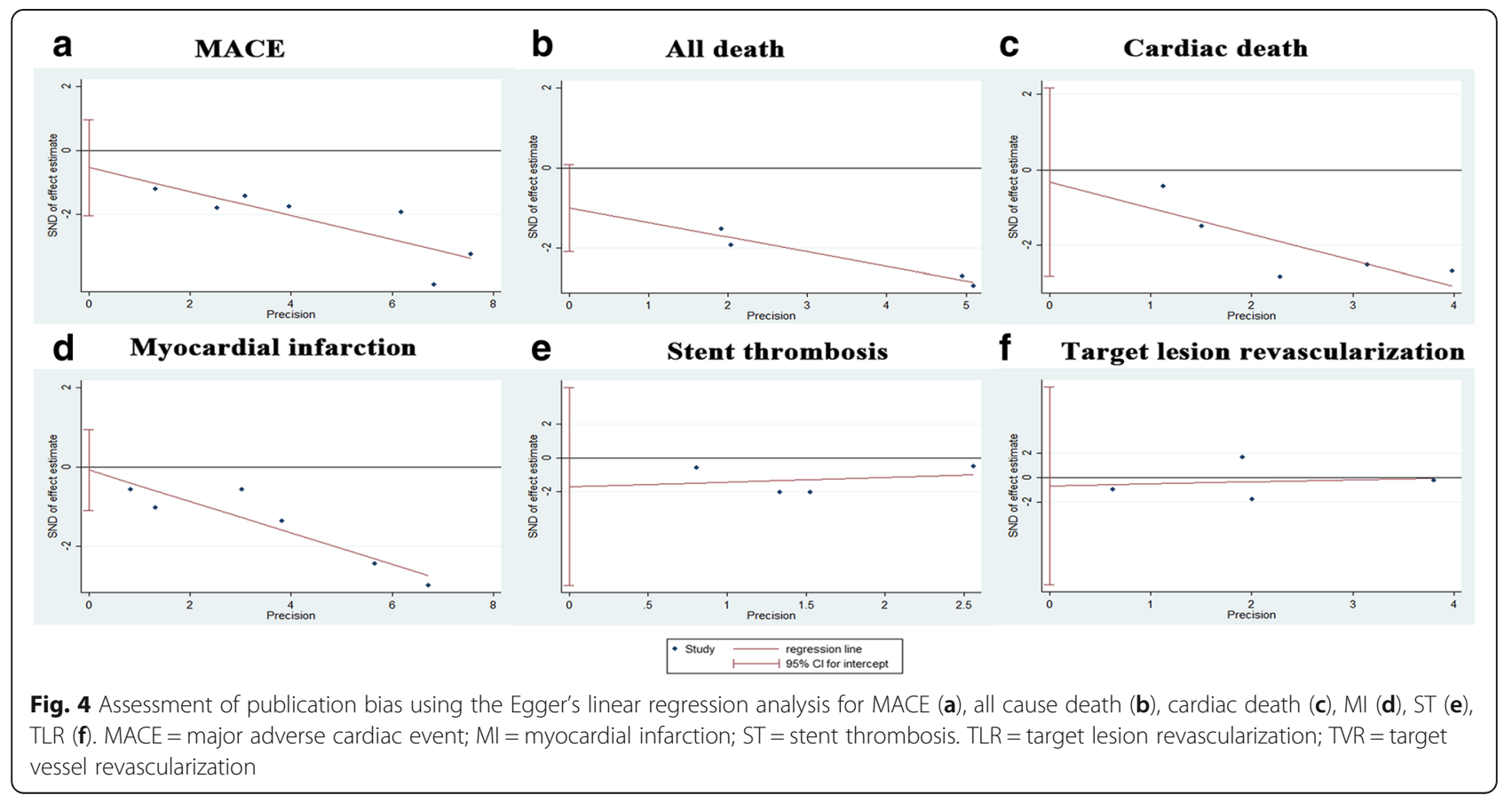


importantly, IVUS can assess stent underexpansion, incomplete lesion coverage (ie, large residual stent edge plaque burden), and malapposition in both LMCA and non-LMCA lesions after DES implantation [36, 37]. Optimal DES implantation is the key to improved patient outcomes. A multivariable logistic regression model of PCI treatment of LMCA lesions showed that IVUS-determined stent underexpansion was an independent predictor for MACE (adjusted hazard ratio $[\mathrm{HR}]=5.56 ; P<0.001$ ); and the two-year MACE-free survival rate was significantly lower in patients with underexpansion of at least one segment versus lesions with no underexpansion $(90 \%$ vs $98 \% ; P<0.001)$ [37].

The current meta-analysis demonstrating that IVUSguided DES implantation led to a significant reduction in the incidence of MACE compared with angiography guidance. This has also been seen in several abstracts at major meetings, and in LMCA subset analyses of larger IVUS versus angiography-guided DES implantation studies. For example, a recently published summary in WCC_2016 concluded that the risk of MACE was significantly lower in LMCA lesions stented with IVUS guidance $(15 \%)$ vs angiography guidance $(24 \%)\left(\mathrm{X}^{2}=\right.$ 42.76; $P=0.009$ ). In addition, the subset analyses of LMCA patients in the ADAPT-DES study also showed a trend toward a reduction in MACE (HR 0.54; 95\% CI $0.23-1.26 ; P=0.15$ ) [30].

The beneficial results of IVUS-guidance demonstrated in the current meta-analysis were mainly related to the lower risk of death and MI. In addition, the use of IVUS was associated with a lower ST risk than reliance on angiography. Considering that ST within LMCA stents may present as sudden death, the incidence of this complication may have been underestimated, but was likely captured in the assessment of patient mortality and/or MI [22].

However, we found that IVUS guidance did not reduce TLR or TVR. This may be attributed to the fact that underpowered study population, low incidence of events and the discretion of the operator who might prefer IVUS guidance for lesions with more complex coronary anatomy.

\section{Limitations}

This meta-analysis had the following limitations. Firstly, only seven studies were included in our meta-analysis; and only one was an (admittedly relatively small) RCT. Indeed, observational studies have significant limitations with selection and ascertainment bias, which influenced the quality of the evidence across studies. In the present meta-analysis, four of six observational studies included propensity score matching to reduce selection biases. Moreover, IVUS-guided DES implantation was still associated with a significant reduction in MACE after sensitivity analysis was performed. The result indicated that IVUS was worthy of being recommended while performing DES placement for LMCA. However, adequately powered, large-scale RCT studies are needed. Secondly, the location of LMCA lesions, the numbers of other diseased vessels, DES type, and specific treatment strategies may also have impacted the clinical outcomes; but most studies did not provide this detailed data so that subgroup analyses could not be conducted. Thirdly, this meta-analysis lacked the power to detect meaningful differences in TLR and TVR due to little sample size and significant heterogeneities.

\section{Conclusions}

The current meta-analysis and systematic review of the literature demonstrate potential clinical value of IVUS in guiding DES implantation for LMCA patients by a significantly reduced risk of MACE, all-cause death, cardiac death, MI, and ST compared with angiography guidance alone. Future studies are warranted as more data becoming available.

\section{Abbreviations \\ ACC: American College of Cardiology; AHA: Angioplasty Summit, American Heart Association; Cl: Confidence interval; DES: Drug eluting stent; FFR: Fractional flow reserve; HR: Hazard ratio; IVUS: Intravascular ultrasound; MACE: Major adverse cardiac event; MI: Myocardial infarction; NCDR: National Cardiovascular Data Registry; NOS: Newcastle-Ottawa scale; PCl: Percutaneous coronary interventions; PES: Paclitaxel eluting stent; RCTs: Randomized control trials; RRs: Relative ratios; SES: Sirolimus eluting stent; ST: Stent thrombosis; TCT: Transcatheter Cardiovascular Therapeutics; TLR: Target lesion revascularization; TVR: Target vessel revascularization; WCC: World Congress of Cardiology}

\section{Funding}

This study was supported by grants for National Natural Science Foundations of China (NSFC, Grant No. 81470492 and 81670317) and Beijing Administration of Foreign Experts Affairs (GDJ-20171100001).

Availability of data and materials

All data generated or analyzed during this study are included in this published article.

\section{Authors' contributions}

YW, XFW and ZCG designed the study; YW, ZCG and YQ acquired, analyzed, and interpreted data; YW and MRL drafted the manuscript; YW and YQ did the literature search and study selection procedures; MSG and XFW did critical revisions for important; All authors read and approved the final manuscript.

\section{Authors' information}

Yue Wang (first author) is a postgraduate student of grade 2016; Yue Wang (fifth author) is a postgraduate student of grade 2017.

Ethics approval and consent to participate Not applicable

\section{Competing interests}

Dr. Mintz receives grant support and/or honoraria from Boston Scientific, Volcano, and ACIST. The other authors have no potential conflicts of interest.

\section{Publisher's Note}

Springer Nature remains neutral with regard to jurisdictional claims in published maps and institutional affiliations. 


\section{Author details}

'Department of Cardiology, Beijing Anzhen Hospital, Capital Medical University, Beijing 100029, China. ${ }^{2}$ Cardiovascular Research Foundation, New York, NY, USA. ${ }^{3}$ Department of Pharmacy, Renji Hospital, School of Medicine, Shanghai Jiao Tong University, Shanghai 200127, China. ${ }^{4}$ Department of Epidemiology, The Key Laboratory of Remodeling-Related Cardiovascular Diseases, Ministry of Education, Beijing Institute of Heart, Lung and Blood Vessel Diseases, Beijing Anzhen Hospital, Capital Medical University, Beijing, China.

Received: 30 March 2018 Accepted: 21 May 2018 Published online: 14 June 2018

\section{References}

1. McDaniel MC, Eshtehardi P, Sawaya FJ, Douglas Jr JS, Samady H. Contemporary clinical applications of coronary intravascular ultrasound. JACC CardiovasC Interv. 2011:4:1155-67.

2. Park SJ, Hong MK, Lee CW, et al. Elective stenting of unprotected left main coronary artery stenosis: effect of debulking before stenting and intravascular ultrasound guidance. J Am Coll Cardiol. 2001;38:1054-60.

3. Kang SJ, Yang DH, Kweon J, et al. Better diagnosis of functionally significant intermediate sized narrowings using intravascular ultrasound-minimal lumen area and coronary computed tomographic angiography-based myocardial segmentation. Am J Cardiol. 2016;117:1282-8.

4. Saito S, Maehara A, Yakushiji T, et al. Serial intravascular ultrasound findings after treatment of chronic total occlusions using drug-eluting stents. Am J Cardiol. 2016;117:727-34

5. Kang SJ, Ahn JM, Kim WJ, et al. Intravascular ultrasound assessment of drugeluting stent coverage of the coronary ostium and effect on outcomes. Am J Cardiol. 2013;111:1401-7.

6. Parise H, Maehara A, Stone GW, Leon MB, Mintz GS. Meta-analysis of randomized studies comparing intravascular ultrasound versus angiographic guidance of percutaneous coronary intervention in pre-drug-eluting stent era. Am J Cardiol. 2011;107:374-82

7. Ahn JM, Kang SJ, Yoon SH, et al. Meta-analysis of outcomes after intravascular ultrasound-guided versus angiography-guided drug-eluting stent implantation in 26,503 patients enrolled in three randomized trials and 14 observational studies. Am J Cardiol. 2014;113:1338-47.

8. Zhang YJ, Faroog V, García-García HM, et al. Comparison of intravascular ultrasound versus angiography-guided drug-eluting stent implantation: a meta-analysis of one randomised trial and ten observational studies involving 19,619 patients. Eur Secur. 2012;8:55-65.

9. Zhang YJ, Pang S, Chen XY, et al. Comparison of intravascular ultrasound guided versus angiography guided drug eluting stent implantation: a systematic review and meta-analysis. BMC Cardiovasc Disord. 2015;15:153.

10. Klersy C, Ferlini M, Raisaro A, et al. Use of IVUS guided coronary stenting with drug eluting stent. A systematic reviewandmeta-analysis of randomized controlled clinical trials and high quality observational studies. Int J Cardiol. 2013;170:54-63.

11. Steinvil A, Zhang YJ, Lee SY, et al. Intravascular ultrasound-guided drugeluting stent implantation: an updated meta-analysis of randomized control trials and observational studies. Int J Cardiol. 2016:216:133-9.

12. Jang JS, Song YJ, Kang W, et al. Intravascular ultrasound-guided implantation of drug-eluting stents to improve outcome a meta-analysis. JACC CardiovasC Interv. 2014;7:233-43.

13. Elgendy IY, Mahmoud AN, Elgendy AY, Bavry AA. Outcomes with intravascular ultrasound-guided stent implantation a meta-analysis of randomized trials in the era of drug-eluting stents. Circ Cardiovasc Interv. 2016;9:e003700.

14. Stone GW, Sabik JF, Serruys PW, et al. Everolimus-eluting stents or bypass surgery for left main coronary artery disease. N Engl J Med. 2016;375:2223-5.

15. Mäkikallio T, Holm NR, Lindsay $M$, et al. Percutaneous coronary angioplasty versus coronary artery bypass grafting in treatment of unprotected left main stenosis (NOBLE): a prospective, randomised, open-label, noninferiority trial. Lancet. 2016;388:2743-52.

16. Tian J, Guan C, Wang W, et al. Intravascular ultrasound guidance improves the long-term prognosis in patients with unprotected left main coronary artery disease undergoing percutaneous coronary intervention. Sci Rep. 2017;7:2377.

17. Laskey WK, Yancy CW, Maisel WH. Thrombosis in coronary drug eluting stents: report from the meeting of the circulatory system medical devices advisory panel of the Food and Drug Administration Center for devices and radiologic health, December 7-8, 2006. Circulation. 2007:115:2352-7.

18. Higgins JP, Altman DG, Gotzsche PC, et al. The Cochrane Collaboration's tool for assessing risk of bias in randomised trials. BMJ. 2011;343:d5928.

19. Wells GA, Shea B, O'Connell D, Tugwell P. The Newcastle-Ottawa Scale (NOS) for assessing the quality if nonrandomized studies in meta-analyses Available at: http://www.ohri.ca/programs/clinical_epidemiology/oxford.htm. Accessed 19, Oct 2009

20. Cheng J, Gao J, Shuai X, Wang G, Tao K. Two-dimensional versus threedimensional laparoscopy in surgical efficacy: a systematic review and metaanalysis. Oncotarget. 2016;7:70979-90.

21. Agostoni P, Valgimigli M, Van Mieghem CA, et al. Comparison of early outcome of percutaneous coronary intervention for unprotected left main coronary artery disease in the drug-eluting stent era with versus without intravascular ultrasonic guidance. Am J Cardiol. 2005:95:644-7.

22. Park SJ, Kim YH, Park DW, et al. Impact of intravascular ultrasound guidance on long-term mortality in stenting for unprotected left main coronary artery stenosis. Circ Cardiovasc Interv. 2009;2:167-77.

23. De La Torre Hernandez JM, Baz Alonso JA, Gomez Hospital JA, et al. Clinical impact of intravascular ultrasound guidance in drug-eluting stent implantation for unprotected left main coronary disease pooled analysis at the patient-level of 4 registries. JACC Cardiovasc Interv. 2014:7:244-54.

24. Gao XF, Kan J, Zhang YJ, et al. Comparison of one-year clinical outcomes between intravascular ultrasound-guided versus angiography-guided implantation of drug-eluting stents for left main lesions: a single-center analysis of a 1,016-patient cohort. Patient Prefer Adherence. 2014;8:1299-309.

25. Tan Q, Wang Q, Liu D, Zhang S, Zhang Y, Li Y. Intravascular ultrasoundguided unprotected left main coronary artery stenting in the elderly. Saudi Med J. 2015;36:549-53.

26. Kim YH, Her AY, Rha SW, et al. Three-year major clinical outcomes of angiography-guided single stenting technique in non-complex left main coronary artery diseases. Int Heart J. 2017;58:704-13.

27. Witzenbichler B, Maehara A, Weisz G, et al. Relationship between intravascular ultrasound guidance and clinical outcomes after drug-eluting stents the assessment of dual antiplatelet therapy with drug-eluting stents (ADAPT-DES) study. Circulation. 2014;129:463-70.

28. Ragosta M. Left main coronary artery disease: importance, diagnosis, assessment, and management. Curr Prob Cardiol. 2015;40:93-126.

29. Chakrabarti AK, Grau-Sepulveda MV, O'Brien S, et al. Angiographic validation of the american college of cardiology foundation-the society of thoracic surgeons collaboration on the comparative effectiveness of revascularization strategies study. Circ Cardiovasc Interv. 2014;7:11-8.

30. Hamilos M, Muller O, Cuisset T, et al. Long-term clinical outcome after fractional flow reserve-guided treatment in patients with angiographically equivocal left main coronary artery stenosis. Circulation. 2009;120:1505-12.

31. Sano K, Mintz GS, Carlier SG, et al. Assessing intermediate left main coronary lesions using intravascular ultrasound. Am Heart J. 2007;154:983-8.

32. Jasti V, Ivan E, Yalamanchili V, Wongpraparut N, Leesar MA. Correlations between fractional flow reserve and intravascular ultrasound in patients with an ambiguous left main coronary artery stenosis. Circulation. 2004;110:2831-6.

33. De la Torre Hernandez JM, Hernandez Hernandez F, Alfonso F, et al. Prospective application of pre-defined intravascular ultrasound criteria for assessment of intermediate left main coronary artery lesions results from the multicenter LITRO study. J Am Coll Cardiol. 2011:58:351-8.

34. Oviedo C, Maehara A, Mintz GS, et al. Intravascular ultrasound classification of plaque distribution in left main coronary artery bifurcations where is the plaque really located? Circ Cardiovasc Interv. 2010;3:105-12

35. Kang SJ, Ahn JM, Kim WJ, et al. Functional and morphological assessment of side branch after left main coronary artery bifurcation stenting with crossover technique. Catheter Cardiovasc Interv. 2014:83:545-52.

36. Fujii K, Carlier SG, Mintz GS, et al. Stent underexpansion and residual reference segment stenosis are related to stent thrombosis after sirolimus-eluting stent implantation: an intravascular ultrasound study. J Am Coll Cardiol. 2005:45:995-8.

37. Kang SJ, Ahn JM, Song H, et al. Comprehensive intravascular ultrasound assessment of stent area and its impact on restenosis and adverse cardiac events in 403 patients with unprotected left main disease. Circ Cardiovasc Interv. 2011:4:562-9. 